\title{
THE DISTRIBUTION OF 2-SELMER RANKS OF QUADRATIC TWISTS OF ELLIPTIC CURVES WITH PARTIAL TWO-TORSION
}

\author{
ZEV KLAGSBRUN AND ROBERT J. LEMKE OLIVER
}

\begin{abstract}
This paper presents a new result concerning the distribution of 2-Selmer ranks in the quadratic twist family of an elliptic curve over an arbitrary number field $K$ with a single point of order two that does not have a cyclic 4-isogeny defined over its twodivision field. We prove that at least half of all the quadratic twists of such an elliptic curve have arbitrarily large 2-Selmer rank, showing that the distribution of 2-Selmer ranks in the quadratic twist family of such an elliptic curve differs from the distribution of 2-Selmer ranks in the quadratic twist family of an elliptic curve having either no rational two-torsion or full rational two-torsion.
\end{abstract}

\section{InTRODUCTION}

1.1. Distributions of Selmer Ranks. Let $E$ be an elliptic curve defined over a number field $K$ and let $\operatorname{Sel}_{2}(E / K)$ be its 2-Selmer group (see Section 2 for its definition). We define the 2-Selmer rank of $E / K$, denoted $d_{2}(E / K)$, by

$$
d_{2}(E / K)=\operatorname{dim}_{\mathbb{F}_{2}} \operatorname{Sel}_{2}(E / K)-\operatorname{dim}_{\mathbb{F}_{2}} E(K)[2] .
$$

In 1994, Heath-Brown proved that the 2-Selmer ranks of all of the quadratic twists of the congruent number curve $E / \mathbb{Q}$ given by $y^{2}=x^{3}-x$ had a particularly nice distribution. In particular, he showed that there are explicits constants $\alpha_{0}, \alpha_{1}, \alpha_{2}, \ldots$ summing to one such that

$$
\lim _{X \rightarrow \infty} \frac{\mid\left\{d \text { squarefree }|d|<X: d_{2}\left(E^{d} / \mathbb{Q}\right)=r\right\} \mid}{\mid\{d \text { squarefree }|d|<X\} \mid}=\alpha_{r}
$$

for every $r \in \mathbb{Z}^{\geq 0}$, where $E^{d}$ is the quadratic twist of $E$ by $d$ [HB94]. This result was extended by Swinnerton-Dyer and Kane to all elliptic curves $E$ over $\mathbb{Q}$ with $E(\mathbb{Q})[2] \simeq \mathbb{Z} / 2 \mathbb{Z} \times \mathbb{Z} / 2 \mathbb{Z}$ that do not have a cyclic 4-isogeny defined over $K$ [Kan10], [SD08].

A similar result was obtained by Klagsbrun, Mazur, and Rubin for elliptic curves $E$ over a general number field $K$ with $\operatorname{Gal}(K(E[2]) / K) \simeq \mathcal{S}_{3}$, where squarefree $d$ are replaced by quadratic characters of $K$ and a suitable ordering of all such characters is taken KMR13.

In this work we show that this type of result does not hold when $E(K)[2] \simeq \mathbb{Z} / 2 \mathbb{Z}$. In particular, we prove the following:

Theorem 1.1. For $d \in \mathcal{O}_{K}$, let $\chi_{d}$ be the quadratic character of $K$ that cuts out the extension $K(\sqrt{d})$ and define

$$
C(K, X):=\left\{\chi_{d}:\left|\mathbf{N}_{K / \mathbb{Q}} d\right|<X\right\} .
$$

Let $E$ be an elliptic curve defined over $K$ with $E(K)[2] \simeq \mathbb{Z} / 2 \mathbb{Z}$ that does not have a cyclic isogeny defined over $K(E[2])$. Then for any fixed $r$,

$$
\liminf _{X \rightarrow \infty} \frac{\left|\left\{\chi \in C(K, X): d_{2}\left(E^{\chi} / K\right) \geq r\right\}\right|}{|C(K, X)|} \geq \frac{1}{2}
$$

where $E^{\chi}$ is the quadratic twist of $E$ by any $d \in \mathcal{O}_{K}$ with $\chi_{d}=\chi$. 
In particular, this shows that there is not a distribution function on 2-Selmer ranks within the quadratic twist family of $E$.

Theorem 1.1 is an easy consequence of the following result.

Theorem 1.2. Let $E$ be an elliptic curve defined over $K$ with $E(K)[2] \simeq \mathbb{Z} / 2 \mathbb{Z}$ that does not have a cyclic isogeny defined over $K(E[2])$. Then the normalized distribution

$$
\frac{P_{r}\left(\mathcal{T}\left(E / E^{\prime}\right), X\right)}{\sqrt{\frac{1}{2} \log \log X}}
$$

converges weakly to the Gaussian distribution

$$
G(z)=\frac{1}{\sqrt{2 \pi}} \int_{-\infty}^{z} e^{\frac{-w^{2}}{2}} \mathrm{~d} w
$$

where

$$
P_{r}\left(\mathcal{T}\left(E / E^{\prime}\right), X\right)=\frac{\left|\left\{\chi \in C(K, X): \operatorname{ord}_{2} \mathcal{T}\left(E^{\chi} / E^{\prime \chi}\right) \leq r\right\}\right|}{|C(K, X)|}
$$

for $X \in \mathbb{R}^{+}, r \in \mathbb{Z}^{\geq 0}$, and $\mathcal{T}\left(E^{\chi} / E^{\prime}\right)$ as defined in Section Q.

In turn, Theorem 1.2 follows from a variant of the Erdôs-Kac theorem for quadratic characters of number fields. Let $C(K)$ denote the set of all quadratic characters of $K$. For any $\chi \in C(K)$, we can associate a unique squarefree ideal $D_{\chi}$ to $\chi$ by taking $D_{\chi}$ to be the squarefree part of the ideal $\langle d\rangle$, where $d$ is any element of $\mathcal{O}_{K}$ such that $\chi_{d}=\chi$. We say that a function $f$ on the ideals of $\mathcal{O}_{K}$ is additive if $f\left(\mathfrak{a} \mathfrak{a}^{\prime}\right)=f(\mathfrak{a})+f\left(\mathfrak{a}^{\prime}\right)$ whenever the ideals $\mathfrak{a}$ and $\mathfrak{a}^{\prime}$ are relatively prime; we define $f(\chi)$ for $\chi \in C(K)$ to be $f\left(D_{\chi}\right)$.

To an additive function $f$, we attach quantities $\mu_{f}(X)$ and $\sigma_{f}(X)$, defined to be

$$
\mu_{f}(X):=\sum_{\mathbf{N} \mathfrak{p}<X} \frac{f(\mathfrak{p})}{\mathbf{N} \mathfrak{p}}, \quad \sigma_{f}(X):=\left(\sum_{\mathbf{N} \mathfrak{p}<X} \frac{f(\mathfrak{p})^{2}}{\mathbf{N} \mathfrak{p}}\right)^{1 / 2},
$$

and we prove the following.

Theorem 1.3. Suppose that $f$ is an additive function such that $0 \leq f(\mathfrak{p}) \leq 1$ for every prime $\mathfrak{p}$. If $\sigma_{f}(X) \rightarrow \infty$ as $X \rightarrow \infty$, then

$$
\lim _{X \rightarrow \infty} \frac{\left|\left\{\chi \in C(K, X): f(\chi)-\mu_{f}(X) \leq z \cdot \sigma_{f}(X)\right\}\right|}{|C(K, X)|}=G(z),
$$

In the special case where $K=\mathbb{Q}$, Theorem 1.1 follows from results recently obtained by Xiong about the distribution of $\operatorname{dim}_{\mathbb{F}_{2}} \operatorname{Sel}_{\phi}(E / \mathbb{Q})$ in quadratic twist families [Xio13]. We are able to obtain results over general number fields by applying different methods to the coarser question about the distribution of $\operatorname{ord}_{2} T\left(E / E^{\prime}\right)$ in twist families (see Theorem 1.2).

Remark 1.4. The methods in the paper can easily be adapted to studying cubic twists of the $j$-invariant 0 curve $y^{2}=x^{3}+k$ for any number field $K$ with $K(\sqrt{k}, \sqrt{-3}) / K$ biquadratic. In that case, Theorem 1.1 holds with 2-Selmer rank replaced by 3-Selmer rank and quadratic characters replaced by cubic characters. 
1.2. Layout. We begin in Section 2 by recalling the definitions of the 2-Selmer group and the Selmer groups associated with a 2-isogeny $\phi$ and presenting some of the connections between them. In Section 3, we examine the behavior of the local conditions for the $\phi$ Selmer group under quadratic twist and show how that quantity $\mathcal{T}\left(E^{\chi} / E^{\prime \chi}\right)$ can be related to the value $f(\chi)$ of an additive function $f$ on $C(K)$. Theorem 1.3 is proved in Section 4 and we conclude with the proofs of Theorems 1.1 and 1.2 in Section 5 .

Acknowledgement. The first author would like to express his thanks to Karl Rubin for his helpful comments and suggestions, to Ken Kramer for a series of valuable discussions, and to Michael Rael and Josiah Sugarman for helpful conversations regarding the Erdős-Kac theorem. The first author was supported by NSF grants DMS-0457481, DMS-0757807, and DMS-0838210.

\section{Selmer Groups}

We begin by recalling the definition of the 2-Selmer group. If $E$ is an elliptic curve defined over a field $K$, then $E(K) / 2 E(K)$ maps into $H^{1}(K, E[2])$ via the Kummer map. The following diagram commutes for every place $v$ of $K$, where $\delta$ is the Kummer map.

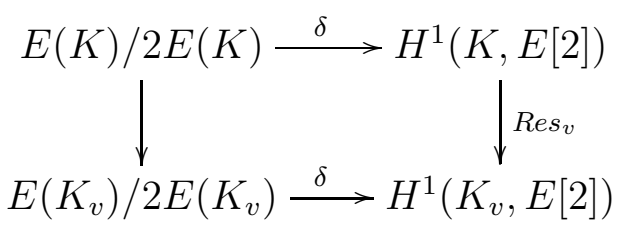

We define a distinguished local subgroup $H_{f}^{1}\left(K_{v}, E[2]\right) \subset H^{1}\left(K_{v}, E[2]\right)$ as the image $\delta\left(E\left(K_{v}\right) / 2 E\left(K_{v}\right)\right) \subset H^{1}\left(K_{v}, E[2]\right)$ for each place $v$ of $K$ and we define the 2-Selmer group of $E / K$, denoted $\operatorname{Sel}_{2}(E / K)$, by

$$
\operatorname{Sel}_{2}(E / K)=\operatorname{ker}\left(H^{1}(K, E[2]) \stackrel{\sum r e s_{v}}{\longrightarrow} \bigoplus_{v \text { of } K} H^{1}\left(K_{v}, E[2]\right) / H_{f}^{1}\left(K_{v}, E[2]\right)\right) .
$$

The 2-Selmer group is a finite dimensional $\mathbb{F}_{2}$-vector space that sits inside the exact sequence of $\mathbb{F}_{2}$-vector spaces

$$
0 \rightarrow E(K) / 2 E(K) \rightarrow \operatorname{Sel}_{2}(E / K) \rightarrow \amalg(E / K)[2] \rightarrow 0
$$

where $\amalg(E / K)$ is the Tate-Shafaravich group of $E$.

If $E(K)$ has a single point of order two, then there is a two-isogeny $\phi: E \rightarrow E^{\prime}$ between $E$ and $E^{\prime}$ with kernel $C=E(K)[2]$. This isogeny gives rise to two Selmer groups.

We have a short exact sequence of $G_{K}$ modules

$$
0 \rightarrow C \rightarrow E(\bar{K}) \stackrel{\phi}{\rightarrow} E^{\prime}(\bar{K}) \rightarrow 0
$$

which gives rise to a long exact sequence of cohomology groups

$$
0 \rightarrow C \rightarrow E(K) \stackrel{\phi}{\rightarrow} E^{\prime}(K) \stackrel{\delta}{\rightarrow} H^{1}(K, C) \rightarrow H^{1}(K, E) \rightarrow H^{1}\left(K, E^{\prime}\right) \ldots
$$

The map $\delta$ is given by $\delta(Q)(\sigma)=\sigma(R)-R$ where $R$ is any point on $E(\bar{K})$ with $\phi(R)=Q$.

This sequence remains exact when we replace $K$ by its completion $K_{v}$ at any place $v$, which gives rise to the following commutative diagram. 


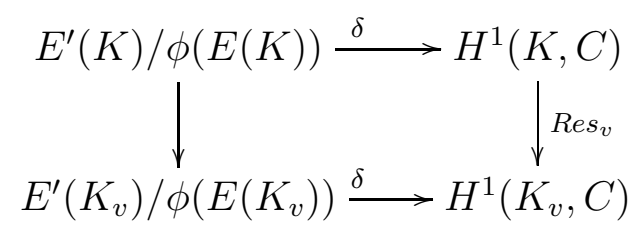

In a manner similar to how we defined the 2-Selmer group, we define distinguished local subgroups $H_{\phi}^{1}\left(K_{v}, C\right) \subset H^{1}\left(K_{v}, C\right)$ as the image of $E^{\prime}\left(K_{v}\right) / \phi\left(E\left(K_{v}\right)\right)$ under $\delta$ for each place $v$ of $K$. We define the $\phi$-Selmer group of $\mathbf{E}$, denoted $\operatorname{Sel}_{\phi}(E / K)$ as

$$
\operatorname{Sel}_{\phi}(E / K)=\operatorname{ker}\left(H^{1}(K, C) \stackrel{\sum r e s_{v}}{\longrightarrow} \bigoplus_{v \text { of } K} H^{1}\left(K_{v}, C\right) / H_{\phi}^{1}\left(K_{v}, C\right)\right) .
$$

The isogeny $\phi$ on $E$ gives gives rise to a dual isogeny $\hat{\phi}$ on $E^{\prime}$ with kernel $C^{\prime}=\phi(E[2])$. Exchanging the roles of $(E, C, \phi)$ and $\left(E^{\prime}, C^{\prime}, \hat{\phi}\right)$ in the above defines the $\hat{\phi}$-Selmer group, $\operatorname{Sel}_{\hat{\phi}}\left(E^{\prime} / K\right)$, as a subgroup of $H^{1}\left(K, C^{\prime}\right)$. The groups $\operatorname{Sel}_{\phi}(E / K)$ and $\operatorname{Sel}_{\hat{\phi}}\left(E^{\prime} / K\right)$ are finite dimensional $\mathbb{F}_{2}$-vector spaces and we can compare the sizes of the $\phi$-Selmer group, the $\hat{\phi}$ Selmer group, and the 2-Selmer group using the following two theorems.

Theorem 2.1. The $\phi$-Selmer group, the $\hat{\phi}$-Selmer group, and the 2-Selmer group sit inside the exact sequence

$$
0 \rightarrow E^{\prime}(K)[2] / \phi(E(K)[2]) \rightarrow \operatorname{Sel}_{\phi}(E / K) \rightarrow \operatorname{Sel}_{2}(E / K) \stackrel{\phi}{\rightarrow} \operatorname{Sel}_{\hat{\phi}}\left(E^{\prime} / K\right) .
$$

Proof. This is a well known diagram chase. See Lemma 2 in [FG08] for example.

The Tamagawa ratio $\mathcal{T}\left(E / E^{\prime}\right)$ defined as $\mathcal{T}\left(E / E^{\prime}\right)=\frac{\left|\operatorname{Sel}_{\phi}(E / K)\right|}{\mid \operatorname{Sel}_{\hat{\phi}}\left(E^{\prime} / K\right)} \mid$ gives a second relationship between the $\mathbb{F}_{2}$-dimensions of $\operatorname{Sel}_{\phi}(E / K)$ and $\operatorname{Sel}_{\hat{\phi}}\left(E^{\prime} / K\right)$.

Theorem 2.2 (Cassels). The Tamagawa ratio $\mathcal{T}\left(E / E^{\prime}\right)$ is given by

$$
\mathcal{T}\left(E / E^{\prime}\right)=\prod_{v \text { of } K} \frac{\left|H_{\phi}^{1}\left(K_{v}, C\right)\right|}{2}
$$

Proof. This is a combination of Theorem 1.1 and equations (1.22) and (3.4) in Cas65.

Stepping back, we observe that if $\mathcal{T}\left(E / E^{\prime}\right) \geq 2^{r+2}$, then $d_{\phi}(E / K) \geq r+2$, and therefore by Theorem 2.1, $d_{2}(E / K) \geq r$. (If $E$ does not have a cyclic 4 -isogeny defined over $K$ then we can in fact show that $\mathcal{T}\left(E / E^{\prime}\right) \geq 2^{r}$ implies that $d_{2}(E / K) \geq r$, but this is entirely unnecessary for our purposes.)

\section{Local Conditions at Twisted Places}

For the remainder of this paper, we will let $E$ be an elliptic curve with $E(K)[2] \simeq \mathbb{Z} / 2 \mathbb{Z}$ and let $\phi: E \rightarrow E^{\prime}$ be the isogeny with kernel $C=E(K)[2]$.

If $\mathfrak{p} \nmid 2$ is a prime where $E$ has good reduction, then $H_{\phi}^{1}\left(K_{\mathfrak{p}}, C\right)$ is a 1 -dimensional $\mathbb{F}_{2^{-}}$ subspace of $H^{1}\left(K_{\mathfrak{p}}, C\right)$ equal to the unramified local subgroup $H_{u}^{1}\left(K_{\mathfrak{p}}, C\right)$. If such a $\mathfrak{p}$ is ramified in the extension $F / K$ cut out by a character $\chi$, then the twisted curve $E^{\chi}$ will have bad reduction at $\mathfrak{p}$. The following lemma addresses the size of $H_{\phi}^{1}\left(K_{\mathfrak{p}}, C^{\chi}\right)$. 
Lemma 3.1. Suppose $\mathfrak{p} \nmid 2$ is a prime where $E$ has good reduction and $\mathfrak{p}$ is ramified in the extension $F / K$ cut out by $\chi$.

(i) If $E\left(K_{\mathfrak{p}}\right)[2] \simeq \mathbb{Z} / 2 \mathbb{Z} \simeq E^{\prime}\left(K_{\mathfrak{p}}\right)[2]$, then $\operatorname{dim}_{\mathbb{F}_{2}} H_{\phi}^{1}\left(K_{\mathfrak{p}}, C^{\chi}\right)=1$.

(ii) If $E\left(K_{\mathfrak{p}}\right)[2] \simeq \mathbb{Z} / 2 \mathbb{Z} \times \mathbb{Z} / 2 \mathbb{Z} \simeq E^{\prime}\left(K_{\mathfrak{p}}\right)[2]$, then $\operatorname{dim}_{\mathbb{F}_{2}} H_{\phi}^{1}\left(K_{\mathfrak{p}}, C^{\chi}\right)=1$.

(iii) If $E\left(K_{\mathfrak{p}}\right)[2] \simeq \mathbb{Z} / 2 \mathbb{Z}$ and $E^{\prime}\left(K_{\mathfrak{p}}\right)[2] \simeq \mathbb{Z} / 2 \mathbb{Z} \times \mathbb{Z} / 2 \mathbb{Z}$, then $\operatorname{dim}_{\mathbb{F}_{2}} H_{\phi}^{1}\left(K_{\mathfrak{p}}, C^{\chi}\right)=2$.

(iv) If $E\left(K_{\mathfrak{p}}\right)[2] \simeq \mathbb{Z} / 2 \mathbb{Z} \times \mathbb{Z} / 2 \mathbb{Z}$ and $E^{\prime}\left(K_{\mathfrak{p}}\right)[2] \simeq \mathbb{Z} / 2 \mathbb{Z}$, then $\operatorname{dim}_{\mathbb{F}_{2}} H_{\phi}^{1}\left(K_{\mathfrak{p}}, C^{\chi}\right)=0$.

Proof. From Lemma 3.7 in [Kla11, we have

$$
E^{\prime \chi}\left(K_{\mathfrak{p}}\right)\left[2^{\infty}\right] / \phi\left(E^{\chi}\left(K_{\mathfrak{p}}\right)\left[2^{\infty}\right]\right)=E^{\chi}\left(K_{\mathfrak{p}}\right)[2] / \phi\left(E^{\chi}\left(K_{\mathfrak{p}}\right)[2]\right) .
$$

All four results then follow immediately.

Remark 3.2. Suppose that $\Delta$ and $\Delta^{\prime}$ are discriminants of any integral models of $E$ and $E^{\prime}$ respectively. The condition that $E\left(K_{\mathfrak{p}}\right)[2] \simeq \mathbb{Z} / 2 \mathbb{Z}\left(\operatorname{resp} . E^{\prime}\left(K_{\mathfrak{p}}\right)[2] \simeq \mathbb{Z} / 2 \mathbb{Z}\right)$ is equivalent to the condition that $\Delta$ (resp. $\Delta^{\prime}$ ) is not a square in $K_{\mathfrak{p}}$. Which case of Lemma 3.1 we are is therefore determined by the Legendre symbols $\left(\frac{\Delta}{\mathfrak{p}}\right)$ and $\left(\frac{\Delta^{\prime}}{\mathfrak{p}}\right)$.

We use Theorem 2.2 and Lemma 3.1 to relate $\operatorname{ord}_{2} \mathcal{T}\left(E^{\chi} / E^{\prime \chi}\right)$ to the value $g(\chi)$ of an additive function $g$ on $C(K)$ defined as follows: For $\chi \in C(K)$ cutting out $F / K$, let

$$
g(\chi)=\sum_{\substack{\mathfrak{p} \text { ramified in } F / K \\ \mathfrak{p} \nmid 2 \Delta \infty}} \frac{\left(\frac{\Delta^{\prime}}{\mathfrak{p}}\right)-\left(\frac{\Delta}{\mathfrak{p}}\right)}{2}
$$

That is, $g(\chi)$ roughly counts the difference between the number of primes ramified in $F / K$ where condition (iii) of Proposition 3.1 is satisfied and the number of primes ramified in $F / K$ where condition $(i v)$ is satisfied. We then have the following:

Proposition 3.3. The order of 2 in the Tamagawa ratio $\mathcal{T}\left(E^{\chi} / E^{\prime \chi}\right)$ is given by

$$
\operatorname{ord}_{2} \mathcal{T}\left(E^{\chi} / E^{\prime \chi}\right)=g(\chi)+\sum_{v \mid 2 \Delta \infty}\left(\operatorname{dim}_{\mathbb{F}_{2}} H_{\phi}^{1}\left(K_{v}, C^{\chi}\right)-1\right) .
$$

Proof. By Thereom 2.2, $\operatorname{ord}_{2} \mathcal{T}\left(E^{\chi} / E^{\prime}\right)$ is given by

$$
\operatorname{ord}_{2} \mathcal{T}\left(E^{\chi} / E^{\prime \chi}\right)=\sum_{v \mid 2 \Delta \Delta_{F / K} \infty}\left(\operatorname{dim}_{\mathbb{F}_{2}} H_{\phi}^{1}\left(K_{v}, C^{d}\right)-1\right)
$$

where $\Delta_{F / K}$ is the relative discriminant of the extension $F / K$ cut out by $\chi$. By Remark 3.2, Lemma 3.1 gives us that

$$
\operatorname{dim}_{\mathbb{F}_{2}} H_{\phi}^{1}\left(K_{\mathfrak{p}}, C^{\chi}\right)-1=\frac{\left(\frac{\Delta^{\prime}}{\mathfrak{p}}\right)-\left(\frac{\Delta}{\mathfrak{p}}\right)}{2}
$$

for places $\mathfrak{p} \mid \Delta_{F / K}$ with $\mathfrak{p} \nmid 2 \Delta \infty$ and the result follows.

\section{The Erdôs-Kac Theorem For Quadratic Characters}

Because the sum

$$
\sum_{v \mid 2 \Delta \infty}\left(\operatorname{dim}_{\mathbb{F}_{2}} H_{\phi}^{1}\left(K_{v}, C^{\chi}\right)-1\right)
$$


can be bounded uniformly for a fixed elliptic curve, Proposition 3.3 suggests that we should study the distribution of the additive function $g(\chi)$ in order to understand the distribution of $\mathcal{T}\left(E^{\chi} / E^{\prime}\right)$ as $\chi$ varies.

When $f: \mathbb{N} \rightarrow \mathbb{C}$ is an additive function on the integers, then under mild hypotheses, the classical Erdôs-Kac Theorem tells us that the distribution of $f$ on natural numbers less than $X$ approaches a normal distribution with mean $\mu(X)$ and variance $\sigma^{2}(X)$ as $X \rightarrow \infty$, where $\mu(X)$ and $\sigma(X)$ are the rational analogues of $\mu_{f}(X)$ and $\sigma_{f}(X)$ defined in the introduction. Theorem 1.3 is the statement that the same type of result holds for additive functions on quadratic characters of a number field.

We begin by observing that if $\chi_{d_{1}}=\chi_{d_{2}}$, then, if $\left(d_{1}\right)=\mathfrak{a b}_{1}^{2}$ and $\left(d_{2}\right)=\mathfrak{a} \mathfrak{b}_{2}^{2}$, $\mathfrak{b}_{1}$ and $\mathfrak{b}_{2}$ lie in the same ideal class of $\mathcal{O}_{K}$. Conversely, if $\mathfrak{b}_{1}$ and $\mathfrak{b}_{2}$ lie in the same class, then $\chi_{d_{1}}=\chi_{\varepsilon d_{2}}$ for some $\varepsilon \in \mathcal{O}_{K}^{\times}$. Thus, we see that elements of $C(K, X)$ correspond to triples $(\mathfrak{b}, \mathfrak{a}, \varepsilon)$ with $\mathfrak{b}$ a representative of its ideal class of minimal norm, $\mathfrak{a}$ a squarefree ideal of norm $\mathbf{N a}<X / \mathbf{N b}^{2}$ such that $\mathfrak{a b}^{2}$ is principal, and $\varepsilon$ an element of $\mathcal{O}_{K}^{\times} /\left(\mathcal{O}_{K}^{\times}\right)^{2}$. From this discussion, it is apparent, for a prime ideal $\mathfrak{p}$ and a fixed choice $\mathfrak{b}_{0}$ of $\mathfrak{b}$, that

$$
\begin{aligned}
\lim _{X \rightarrow \infty} \frac{\left|\left\{\chi \in C(K, X) \leftrightarrow\left(\mathfrak{b}_{0}, \mathfrak{a}, \varepsilon\right): \mathfrak{p} \mid \mathfrak{a}\right\}\right|}{|C(K, X)|} & =\operatorname{Pr}\left(\mathfrak{p} \mid \mathfrak{a}: \mathfrak{a} \text { is squarefree, } \mathfrak{a} \mathfrak{b}_{0}^{2} \text { is principal }\right) \\
& =\frac{1}{\mathbf{N p}+1},
\end{aligned}
$$

whence, ranging over all $\chi$, the probablity that $\mathfrak{p} \mid \mathfrak{a}$ is $\frac{1}{\mathbf{N} \mathfrak{p}+1}$.

Thus, treating the events $\mathfrak{p}_{1} \mid \mathfrak{a}$ and $\mathfrak{p}_{2} \mid \mathfrak{a}$ as independent, we might predict that

$$
\frac{1}{|C(K, X)|} \sum_{\chi \in C(K, X)} f(\chi) \approx \tilde{\mu}_{f}(X)
$$

where

$$
\begin{aligned}
\tilde{\mu}_{f}(X) & :=\sum_{\mathbf{N} \mathfrak{p}<X} \frac{f(\mathfrak{p})}{\mathbf{N p}+1} \\
& =\mu_{f}(X)+O(1) .
\end{aligned}
$$

In fact, this prediction is true, which we establish using the method of moments, following the blueprint of Granville and Soundararajan [GS07]. This requires the following technical result about the function $g_{\mathfrak{p}}(\chi)$, defined to be

$$
g_{\mathfrak{p}}(\chi):= \begin{cases}f(\mathfrak{p})\left(1-\frac{1}{\mathbf{N} \mathfrak{p}+1}\right) & \text { if } \mathfrak{p} \mid \mathfrak{a}, \text { and } \\ f(\mathfrak{p})\left(-\frac{1}{\mathbf{N} \mathfrak{p}+1}\right) & \text { if } \mathfrak{p} \nmid \mathfrak{a} .\end{cases}
$$

Theorem 4.1. With notation as above, uniformly for $k \leq \sigma_{f}(z)^{2 / 3}$, we have that

$$
\frac{1}{|C(K, X)|} \sum_{\chi \in C(K, X)}\left(\sum_{\mathbf{N} \mathfrak{p}<z} g_{\mathfrak{p}}(\chi)\right)^{k}=c_{k} \sigma_{f}(z)^{k}\left(1+O\left(\frac{k^{3}}{\sigma_{f}(z)^{2}}\right)\right)+O\left(X^{\lambda-1} 3^{k} \pi_{K}(z)^{k}\right)
$$

if $k$ is even, and

$$
\frac{1}{|C(K, X)|} \sum_{\chi \in C(K, X)}\left(\sum_{\mathbf{N} \mathfrak{p}<z} g_{\mathfrak{p}}(\chi)\right)^{k} \ll c_{k} \sigma_{f}(z)^{k-1} k^{3 / 2}+X^{\lambda-1} 3^{k} \pi_{K}(z)^{k}
$$


if $k$ is odd. Here, $c_{k}=\Gamma(k+1) / 2^{k / 2} \Gamma\left(\frac{k}{2}+1\right)$ and $\lambda<1$ depends only on the degree of $K$.

The proof of Theorem 4.1 relies upon a result about the distribution of squarefree ideals. To this end, given ideals $\mathfrak{c}$ and $\mathfrak{q}$ and squarefree $\mathfrak{d} \mid \mathfrak{q}$, define $N^{\operatorname{sf}}(X ; \mathfrak{c}, \mathfrak{q}, \mathfrak{d})$ to be the number of squarefree ideals $\mathfrak{a}$ of norm up to $X$ in the same class as $\mathfrak{c}$ and such that $(\mathfrak{a}, \mathfrak{q})=\mathfrak{d}$. We then have:

Lemma 4.2. With notation as above, we have that

$$
N^{\mathrm{sf}}(X ; \mathfrak{c}, \mathfrak{q}, \mathfrak{d})=\frac{1}{|\mathrm{Cl}(K)|} \frac{\operatorname{res}_{s=1} \zeta_{K}(s)}{\zeta_{K}(2)} \phi(\mathfrak{q}, \mathfrak{d}) X+O\left(X^{\lambda} 3^{\omega(\mathfrak{q})}\right)
$$

where $\lambda=\frac{\operatorname{deg} K-1}{\operatorname{deg} K+1}$ if $\operatorname{deg} K \geq 3$ and $\lambda=1 / 2$ otherwise, $\omega(\mathfrak{q})$ denotes the number of distinct primes dividing $\mathfrak{q}$, and

$$
\phi(\mathfrak{q}, \mathfrak{d})=\prod_{\mathfrak{p} \mid \mathfrak{d}} \frac{1}{\mathbf{N} \mathfrak{p}+1} \prod_{\mathfrak{p} \mid \mathfrak{q}, \mathfrak{p} \nmid \mathfrak{d}} \frac{\mathrm{Np}}{\mathbf{N p}+1} .
$$

Proof. This follows from elementary considerations and the classical estimate

$$
\sum_{\substack{\mathbf{N a}<X \\ \mathfrak{a c}^{-1} \text { prin. }}} 1=\frac{1}{|\mathrm{Cl}(K)|} \operatorname{res}_{s=1} \zeta_{K}(s) \cdot X+O\left(X^{\frac{\operatorname{deg} K-1}{\operatorname{deg} K+1}}\right) .
$$

Proof of Theorem 4.1. For any ideal $\mathfrak{q}$, define $g_{\mathfrak{q}}(\chi):=\prod_{\mathfrak{p}^{\alpha} \| \mathfrak{q}} g_{\mathfrak{p}}(\chi)^{\alpha}$. We then have that

$$
\begin{aligned}
\sum_{\chi \in C(K, X)}\left(\sum_{\mathbf{N} \mathfrak{p}<z} g_{\mathfrak{p}}(\chi)\right)^{k} & =\left|\mathcal{O}_{K}^{\times} /\left(\mathcal{O}_{K}^{\times}\right)^{2}\right| \cdot \sum_{\mathfrak{b}} \sum_{\substack{\mathbf{N a}<\frac{X}{\mathbf{N b}^{2}} \\
\mathfrak{a} \mathfrak{b}^{2} \text { prin. }}}^{\prime}\left(\sum_{\mathbf{N} \mathfrak{p}<z} g_{\mathfrak{p}}(\mathfrak{a})\right)^{k} \\
& =\left|\mathcal{O}_{K}^{\times} /\left(\mathcal{O}_{K}^{\times}\right)^{2}\right| \cdot \sum_{\mathfrak{b}} \sum_{\substack { \mathbf{N} \mathfrak{p}_{1}, \ldots, \mathbf{N p}_{k}<z \\
\begin{subarray}{c}{\mathbf{N a}<\frac{X}{\mathbf{N}^{2}} \\
\mathfrak{a} \mathfrak{b}^{2} \text { principal }{ \mathbf { N } \mathfrak { p } _ { 1 } , \ldots , \mathbf { N p } _ { k } < z \\
\begin{subarray} { c } { \mathbf { N a } < \frac { X } { \mathbf { N } ^ { 2 } } \\
\mathfrak { a } \mathfrak { b } ^ { 2 } \text { principal } } }\end{subarray}}^{\prime} g_{\mathfrak{p}_{1} \ldots \mathfrak{p}_{k}}(\mathfrak{a}),
\end{aligned}
$$

where, as expected, the summation over $\mathfrak{b}$ is taken to be over representatives of minimal norm for each ideal class and the prime on the summation over $\mathfrak{a}$ indicates it is to be taken over squarefree ideals. Given an ideal $\mathfrak{c}$, we now consider for any $\mathfrak{q}$ the more general summation

$$
\begin{aligned}
\sum_{\substack{\mathbf{N} \mathfrak{a}<Y \\
\mathfrak{a}^{-1} \text { principal }}}^{\prime} g_{\mathfrak{q}}(\mathfrak{a}) & =\sum_{\mathfrak{d} \mid \sqrt{\mathfrak{q}}} g_{\mathfrak{q}}(\mathfrak{d}) N^{\mathrm{sf}}(Y ; \mathfrak{c}, \mathfrak{q}, \mathfrak{d}) \\
& =\frac{Y}{|\mathrm{Cl}(K)|} \frac{\operatorname{res}_{s=1} \zeta_{K}(s)}{\zeta_{K}(2)} \sum_{\mathfrak{d} \mid \sqrt{\mathfrak{q}}} g_{\mathfrak{q}}(\mathfrak{d}) \phi(\mathfrak{q}, \mathfrak{d})+O\left(Y^{\lambda} 3^{\omega(\mathfrak{q})} \sum_{\mathfrak{d} \mid \sqrt{\mathfrak{q}}}\left|g_{\mathfrak{q}}(\mathfrak{d})\right|\right), \\
& =: \frac{Y}{|\mathrm{Cl}(K)|} \frac{\operatorname{res}_{s=1} \zeta_{K}(s)}{\zeta_{K}(2)} G(\mathfrak{q})+O\left(Y^{\lambda} 3^{\omega(\mathfrak{q})}\right)
\end{aligned}
$$

say, where $\sqrt{\mathfrak{q}}=\prod_{\mathfrak{p} \mid \mathfrak{q}} \mathfrak{p}$. We note that $G(\mathfrak{q})$ is multiplicative, and is given by

$$
G(\mathfrak{q})=\prod_{\mathfrak{p}^{\alpha} \| \mathfrak{q}} \frac{f(\mathfrak{p})^{\alpha}}{\mathbf{N} \mathfrak{p}+1}\left(\left(1-\frac{1}{\mathbf{N} \mathfrak{p}+1}\right)^{\alpha}+\mathbf{N} \mathfrak{p} \cdot\left(-\frac{1}{\mathbf{N p}+1}\right)^{\alpha}\right)
$$


Thus, $G(\mathfrak{q})=0$ unless each $\alpha$ is at least two, i.e. $\mathfrak{q}$ is square-full.

Returning to the original problem, we find that

$$
\sum_{\chi \in C(K, X)}\left(\sum_{\mathbf{N} \mathfrak{p}<z} g_{\mathfrak{p}}(\chi)\right)^{k}=c(K) \cdot X \sum_{\mathbf{N p}_{1}, \ldots, \mathbf{N p}_{k}<z} G\left(\mathfrak{p}_{1} \ldots \mathfrak{p}_{k}\right)+O\left(X^{\lambda} 3^{k} \pi_{K}(z)^{k}\right),
$$

where $\pi_{K}(z):=\#\{\mathfrak{p}: \mathbf{N p}<z\}$ and

$$
c(K):=\left|\mathcal{O}_{K}^{\times} /\left(\mathcal{O}_{K}^{\times}\right)^{2}\right| \frac{1}{|\mathrm{Cl}(K)|} \frac{\operatorname{res}_{s=1} \zeta_{K}(s)}{\zeta_{K}(2)} \sum_{\mathfrak{b}} \frac{1}{\mathbf{N b}^{2}} .
$$

Noting that the above discussion also proves that $|C(K, X)|=c(K) \cdot X+O\left(X^{\lambda}\right)$, the goal is to estimate the summation over $\mathfrak{p}_{1}, \ldots, \mathfrak{p}_{k}$. Since $G(\mathfrak{q})=0$ unless $\mathfrak{q}$ is square-full, we have that

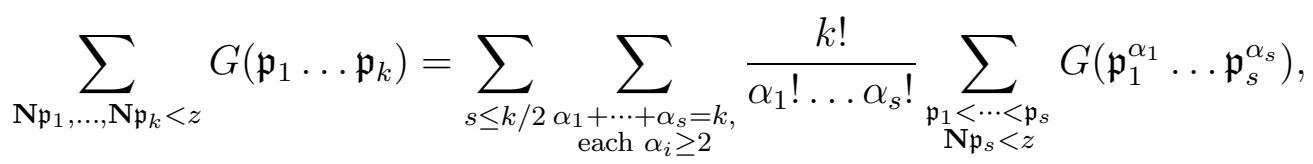

where $\mathfrak{p}<\mathfrak{p}^{\prime}$ is determined by a norm-compatible linear ordering on the prime ideals of $\mathcal{O}_{K}$. We note that, since $G\left(\mathfrak{p}^{\alpha}\right) \leq \frac{f(\mathfrak{p})^{2}}{N \mathfrak{p}}$, the inner summation contributes no more than $O\left(\sigma_{f}(z)^{2 s}\right)$, which will be an error term unless $s=\frac{k}{2}$; the dependence of this error on $k$ can be sussed out exactly as in Granville and Soundararajan's work. In fact, the handling of the main term, arising when $k$ is even and $s=\frac{k}{2}$, is also essentially the same. In particular, the inner summation is equal to

$$
\begin{aligned}
\frac{1}{\left(\frac{k}{2}\right) !} \sum_{\substack{\mathbf{N} \mathfrak{p}_{1}, \ldots, \mathbf{N p}_{\frac{k}{2}}<z \\
\text { distinct }}} G\left(\mathfrak{p}_{1}^{2} \ldots \mathfrak{p}_{\frac{k}{2}}^{2}\right) & =\frac{1}{\left(\frac{k}{2}\right) !}\left(\sum_{\mathbf{N} \mathfrak{p}<z} G\left(\mathfrak{p}^{2}\right)+O(\log \log k)\right)^{k / 2} \\
& =\frac{1}{\left(\frac{k}{2}\right) !}\left(\sigma_{f}(z)^{2}+O(\log \log k)\right)^{k / 2}
\end{aligned}
$$

This yields Theorem 4.1,

We are now ready to prove Theorem [1.3,

Proof of Theorem 1.3. Recall that we wish to show that the quantity

$$
\frac{f(\chi)-\mu_{f}(X)}{\sigma_{f}(X)}, \chi \in C(K, X),
$$

is normally distributed as $X \rightarrow \infty$. As remarked above, we will do so using the method of moments. In particular, we have that

$$
\begin{aligned}
\frac{1}{|C(K, X)|} \sum_{\chi \in C(K, X)}\left(f(\chi)-\mu_{f}(X)\right)^{k} & =\frac{1}{|C(K, X)|} \sum_{\chi \in C(K, X)}\left(\sum_{\mathfrak{p} \mid \mathfrak{a}} f(\mathfrak{p})-\sum_{\mathbf{N} \mathfrak{p}<X} \frac{f(\mathfrak{p})}{\mathbf{N} \mathfrak{p}+1}+O(1)\right)^{k} \\
& =\frac{1}{|C(K, X)|} \sum_{\chi \in C(K, X)}\left(\sum_{\mathbf{N} \mathfrak{p}<z} g_{\mathfrak{p}}(\mathfrak{a})+O\left(\frac{\log X}{\log z}\right)\right)^{k} .
\end{aligned}
$$


Considering the error term in Theorem 4.1, we take $z=X^{\frac{1-\lambda}{k}}$. With this choice, the inner summation becomes

$$
\left(\sum_{\mathbf{N} \mathfrak{p}<z} g_{\mathfrak{p}}(\mathfrak{a})\right)^{k}+O\left(\sum_{j=0}^{k-1} k^{j}\left|\sum_{\mathbf{N} \mathfrak{p}<z} g_{\mathfrak{p}}(\mathfrak{a})\right|^{k-j}\right)
$$

hence Theorem 4.1, the Cauchy-Schwarz inequality, and the fact that $\sigma_{f}(z)=\sigma_{f}(X)+$ $O(\log k)$ yield that

$$
\frac{1}{|C(K, X)|} \sum_{\chi \in C(K, X)}\left(f(\chi)-\mu_{f}(X)\right)^{k}=c_{k} \sigma_{f}(X)^{k}\left(1+O\left(\frac{k^{3 / 2}}{\sigma_{f}(X)}\right)\right)
$$

if $k$ is even, and

$$
\frac{1}{|C(K, X)|} \sum_{\chi \in C(K, X)}\left(f(\chi)-\mu_{f}(X)\right)^{k} \ll c_{k} \sigma_{f}(X)^{k-1} k^{3 / 2}
$$

if $k$ is odd. This proves the theorem.

\section{Proof of Main Theorems}

In order to apply Theorem 1.3 to the additive function $g(\chi)$ defined in (3), we need to evaluate the quantities $\mu_{g}(X)$ and $\sigma_{g}(X)$. We begin with the following

Proposition 5.1. Let $c \in K^{\times}$be non-square. Then

$$
\sum_{\mathbf{N} \mathfrak{p} \leq X} \frac{1+\left(\frac{c}{\mathfrak{p}}\right)}{\mathbf{N p}}=\log \log X+O(1)
$$

Proof. This is a consequence of the prime ideal theorem and the fact that the Hecke $L$ function attached to the non-trivial character of $\operatorname{Gal}(K(\sqrt{c}) / K)$ is analytic and non-vanishing on the line $\Re(s)=1$.

We now decompose $\mu_{g}(X)$ as

$$
\mu_{g}(X)=\frac{1}{2} \sum_{\substack{\mathbf{N} \mathfrak{p}<X \\ p \nmid 2 \Delta \infty}} \frac{1+\left(\frac{\Delta^{\prime}}{\mathfrak{p}}\right)}{\mathrm{Np}}-\frac{1}{2} \sum_{\substack{\mathbf{N} \mathfrak{p}<X \\ \mathfrak{p} \nmid 2 \Delta \infty}} \frac{1+\left(\frac{\Delta}{\mathfrak{p}}\right)}{\mathrm{Np}}
$$

and it immediately follows from Proposition 5.1 that $\mu_{g}(X)=O(1)$. We also rewrite $\sigma_{g}(X)$ as

$$
\sigma_{g}(X)=\left(\sum_{\substack{\mathbf{N} \leq X X, \mathfrak{p} \nmid 2 \Delta \infty \\\left(\frac{\Delta}{\mathfrak{p}}\right) \neq\left(\frac{\Delta^{\prime}}{\mathfrak{p}}\right)}} \frac{1}{\mathbf{N p}}\right)^{1 / 2}=\left(\frac{1}{2} \sum_{\substack{\mathbf{N} \mathfrak{p} \leq X \\ \mathfrak{p} \nmid 2 \Delta \infty}} \frac{1-\left(\frac{\Delta \Delta^{\prime}}{\mathfrak{p}}\right)}{\mathbf{N p}}\right)^{1 / 2} .
$$

In order to apply Proposition 5.1 to $\sigma_{g}(X)$, we therefore need $\Delta \Delta^{\prime}$ to be non-square in $K$. This will be the case when $E$ does not have a cyclic 4-isogeny defined over $K(E[2])$.

Proposition 5.2. If $E$ is an elliptic curve with $E(K)[2] \simeq \mathbb{Z} / 2 \mathbb{Z}$ that does not have a cyclic 4-isogeny defined over $K(E[2])$, then $\Delta \Delta^{\prime} \notin\left(K^{\times}\right)^{2}$ 
Proof. Let $Q^{\prime} \in E^{\prime}[2]-C^{\prime}, C=\langle P\rangle$, and take $Q \in E[4]$ with $\phi(Q)=Q^{\prime}$. Since $Q^{\prime} \in$ $E^{\prime}(K)[2]-C^{\prime}$, and both $\phi \circ \hat{\phi}=[2]_{E^{\prime}}$ and $\hat{\phi} \circ \phi=[2]_{E}$, it follows that $2 Q=\hat{\phi}\left(Q^{\prime}\right)=P$. Let $M=K(E[2])$. Since $E$ has no cyclic 4-isogeny defined over $M$, there exists $\sigma \in G_{M}$ such that $\sigma(Q) \notin\langle Q\rangle=\{0, Q, P, Q+P\}$. In particular, since $\phi^{-1}\left(Q^{\prime}\right) \subset\langle Q\rangle$, we get that $\phi(\sigma(Q)) \neq Q^{\prime}$. We then get that

$$
\sigma\left(Q^{\prime}\right)=\sigma(\phi(Q))=\phi(\sigma(Q)) \neq Q^{\prime}
$$

showing that $Q^{\prime}$ is not defined over $M$, and therefore that $K\left(E^{\prime}[2]\right) \not \subset M$. It then follows that $K(E[2])$ and $K\left(E^{\prime}[2]\right)$ are disjoint quadratic extensions of $K$ and that $E^{\prime}(K)[2] \simeq \mathbb{Z} / 2 \mathbb{Z}$. As $K(E[2])$ and $K\left(E^{\prime}[2]\right)$ are given by $K(\sqrt{\Delta})$ and $K\left(\sqrt{\Delta^{\prime}}\right)$ respectively, it follows that $\Delta \Delta^{\prime} \notin\left(K^{\times}\right)^{2}$.

By Proposition 5.1, we therefore get that $\sigma_{g}(X)=\sqrt{\frac{1}{2} \log \log X}+O(1)$ whenever $E$ does not have a cyclic 4-isogeny defined over $K(E[2])$.

Proof of Theorem 1.2. Applying Theorem 1.3 to $g(\chi)$, we get that

$$
\lim _{X \rightarrow \infty} \frac{\left|\left\{\chi \in C(K, X): g(\chi)-O(1) \leq z\left(\sqrt{\frac{1}{2} \log \log X}+O(1)\right)\right\}\right|}{|C(K, X)|}=G(z)
$$

for every $z \in \mathbb{R}$. By Proposition 3.3, there is some contant $C$, independent of $\chi$, such that $\left|g(\chi)-\operatorname{ord}_{2} \mathcal{T}\left(E^{\chi} / E^{\prime \chi}\right)\right|<C$, so in fact (44) holds with $g(\chi)$ replaced by $\operatorname{ord}_{2} \mathcal{T}\left(E^{\chi} / E^{\prime \chi}\right)$ and the result follows.

Proof of Theorem 1.1. By Theorem 1.2,

$$
\lim _{X \rightarrow \infty} \frac{\left|\left\{\chi \in C(K, X): \operatorname{ord}_{2} \mathcal{T}\left(E^{\chi} / E^{\prime \chi}\right) \geq r\right\}\right|}{|C(K, X)|}=\frac{1}{2}
$$

for any fixed $r \geq 0$. As $d_{2}\left(E^{\chi} / K\right) \geq \operatorname{ord}_{2} \mathcal{T}\left(E^{\chi} / E^{\prime \chi}\right)-2$, this shows that for any $\epsilon>0$,

$$
\frac{\left|\left\{\chi \in C(K, X): d_{2}\left(E^{\chi} / K\right) \geq r\right\}\right|}{|C(K, X)|} \geq \frac{1}{2}-\epsilon
$$

for sufficiently large $X$.

\section{REFERENCES}

[Cas65] J.W.S. Cassels. Arithmetic on curves of genus 1. VIII: On the conjectures of Birch and SwinnertonDyer. Journal für die reine und angewandte Mathematik (Crelles Journal), 1965(217):180-199, 1965.

[FG08] E.V. Flynn and C. Grattoni. Descent via isogeny on elliptic curves with large rational torsion subgroups. Journal of Symbolic Computation, 43(4):293-303, 2008.

[GS07] A. Granville and K. Soundararajan. Sieving and the Erdôs-Kac theorem. Equidistribution in number theory, an introduction, pages 15-27, 2007.

[HB94] D.R. Heath-Brown. The size of Selmer groups for the congruent number problem, II. Inventiones mathematicae, 118(1):331-370, 1994.

[Kan10] D.M. Kane. On the ranks of the 2-Selmer groups of twists of a given elliptic curve. Preprint available at http://arxiv. org/abs/1009.1365, 2010.

[Kla11] Z. Klagsbrun. Selmer ranks of quadratic twists of elliptic curves with partial rational two-torsion. Preprint available at http://arxiv.org/abs/1201.5408, 2011.

[KMR13] Z. Klagsbrun, B Mazur, and K. Rubin. A Markov model for Selmer ranks in families of twists. Preprint available at http://arxiv.org/pdf/1303.650\%v1.pdf, 2013. 
[SD08] P. Swinnerton-Dyer. The effect of twisting on the 2-Selmer group. In Mathematical Proceedings of the Cambridge Philosophical Society, volume 145, pages 513-526. Cambridge Univ Press, 2008.

[Xio13] Maosheng Xiong. On Selmer groups of quadratic twists of elliptic curve a two-torsion over $\mathfrak{q}$. Mathematika, 2013.

E-mail address: zev.klagsbrun@gmail.com

E-mail address: rjlo@stanford.edu 